\title{
Diluted antiferromagnets in a field seem to be in a different universality class than the random-field Ising model
}

\author{
Björn Ahrens, ${ }^{1,2}$ Jianping Xiao, ${ }^{1}$ Alexander K. Hartmann, ${ }^{2}$ and Helmut G. Katzgraber ${ }^{1,3,4}$ \\ ${ }^{1}$ Department of Physics and Astronomy, Texas A $6 M$ University, College Station, Texas 77843-4242, USA \\ ${ }^{2}$ Institut für Physik, Carl-von-Ossietzky Universität, 26111 Oldenburg, Germany \\ ${ }^{3}$ Materials Science and Engineering Program, Texas A $6 M$ University, College Station, TX 77843-3003, USA \\ ${ }^{4}$ Theoretische Physik, ETH Zurich, CH-8093 Zurich, Switzerland
}

(Dated: April 12, 2022)

\begin{abstract}
We perform large-scale Monte Carlo simulations using the Machta-Newman-Chayes algorithms to study the critical behavior of both the diluted antiferromagnet in a field with $30 \%$ dilution and the random-field Ising model with Gaussian random fields for different field strengths. Analytical calculations by Cardy [Phys. Rev. B 29, 505 (1984)] predict that both models map onto each other and share the same universality class in the limit of vanishing fields. However, a detailed finite-size scaling analysis of the Binder cumulant, the two-point finite-size correlation length, and the susceptibility suggests that even in the limit of small fields, where the mapping is expected to work, both models are not in the same universality class. Based on our numerical data, we present analytical expressions for the phase boundaries of both models.
\end{abstract}

PACS numbers: 64.60.De, 75.10.Nr, 75.40.-s,75.50.Lk

\section{INTRODUCTION}

The random-field Ising mode $\mathbf{l}^{\underline{\underline{1}}}$ (RFIM) is of paramount importance in the field of disordered systems $\stackrel{2-5}{-\underline{5}}$ A plethora of problems across disciplines can be studied via the RFIM, ranging from the thermodynamics of disordered magnets $\stackrel{\underline{6}}{\underline{7}}$ hysteresis in magnetic systems and Barkhausen noise, $\stackrel{-\underline{-9}}{\underline{\underline{9}}}$ tunable domain-wall pinning $\stackrel{10}{=}$ the random pinning of polymers,, 11 and even water seepage in porous media. As such, the RFIM is still under intense theoretical, as well as numerical and experimental scrutiny.

More recently, the RFIM has been realized in diluted dipolar magnets in a transverse field such as $\mathrm{LiHo}_{\mathrm{x}} \mathrm{Y}_{1-\mathrm{x}} \mathrm{F}_{4}$. However, most experimental studies focus on diluted antiferromagnets in a field (DAFF), such as $\mathrm{Fe}_{\mathrm{x}} \mathrm{Zn}_{1-\mathrm{x}} \mathrm{F}_{2}, \underline{\underline{3}} \underline{\underline{12}} \underline{\underline{15}}$ Fishman and Aharony $\underline{\underline{16}}$ were the first to note that a random antiferromagnet in a field can be described by the RFIM, and Cardy $\underline{17}$ predicted, using a mean-field argument, that the critical behavior of both models should be in the same universality class in the limit of small fields. The work of Fishman and Aharony,$\underline{16}$ as well as Cardy,$\underline{17}$ therefore opened the door for intense experimental investigation of the RFIM via DAFF materials. However, early experiments and simulations already hinted towards discrepancies between experimental and numerical estimates of the critical exponents $\frac{3,18,19}{19}$ On the other hand, exact groundstate calculations using moderate system sizes suggested an agreement between the critical exponents for both models when the random fields are Gaussian distributed, however not when the random fields are drawn from a bimodal distribution $\stackrel{18,19}{\underline{19}}$ This result, however, has been revised recently $\stackrel{20}{\rightleftharpoons}$ i.e., the universality class of the RFIM is independent of the form of the implemented randomfield distribution.

In this paper we perform detailed Monte Carlo sim- ulations of both the RFIM and the DAFF. The latter is studied at $30 \%$ dilution, i.e., below the percolation threshold for vacancies. Using a finite-size scaling analysis of the Binder cumulant, the two-point finite-size correlation function, and the susceptibility, we show that even in the limit of small fields - where the Cardy mapping 17 is expected to work - both models seem to be in different universality classes. Therefore, care should be taken when making predictions for the critical behavior of the RFIM using experiments on DAFF materials. Finally, we present heuristic analytical expressions based on our numerical data for the phase boundaries of both models to help guide experimental studies.

The manuscript is structured as follows. In Sec. [I] we introduce both the RFIM and the DAFF, followed by an explanation of the used algorithms in Sec. III as well as the measured quantities in Sec. [V] In Sec. D] we show our numerical results, followed by a detailed discussion of the phase boundaries and universality between both models in Sec. VI.

\section{MODELS}

The Hamiltonian of the diluted antiferromagnet in a field (DAFF) is given by

$$
\mathcal{H}_{\mathrm{DAFF}}=+J \sum_{\langle i, j\rangle} \varepsilon_{i} \varepsilon_{j} S_{i} S_{j}-B \sum_{i} \varepsilon_{i} S_{i},
$$

and the Hamiltonian for the random-field Ising model (RFIM) is

$$
\mathcal{H}_{\mathrm{RFIM}}=-J \sum_{\langle i, j\rangle} S_{i} S_{j}-h \sum_{i} \delta_{i} S_{i} .
$$

In Eqs. (11) and (2) $S_{i} \in\{ \pm 1\}$ represent Ising spins, $J=1$ is the coupling constant between two adjacent spins, and 
$\langle i, j\rangle$ denotes a sum over nearest neighbors. The linear term in $S_{i}$ couples to an external field: For the DAFF it is an externally-applied uniform field $B$, whereas for the RFIM the spins couple to a random field of strength $h \delta_{i}$, where the $\delta_{i}$ are quenched random variables chosen from a Gaussian distribution with zero mean and standard deviation unity. This means that the typical field has strength $h$. In the DAFF $\varepsilon_{i} \in\{0,1\}$ represents the site dilution, where each site is randomly and independently occupied by a $\operatorname{spin}\left(\varepsilon_{i}=1\right)$ with probability $p$. Here, we fix the dilution to $1-p=0.3$. Both models are studied in three space dimensions on a lattice with $N=L^{3}$ spins, $L$ being the linear size of the lattice.

\section{ALGORITHM}

The simulations are done using the Machta-NewmanChayes replica-exchange (MNC) algorithm 21 combined with single-spin Metropolis Monte Carlo $\stackrel{22,23}{2}$ The MNC algorithm is a mixture of the Swendsen-Wang exchange algorithm ${ }^{24}$ and simulated tempering Monte Carlo. ${ }^{25.26}$ Note that the latter is not efficient when simulating random-field systems $2 \frac{27}{2}$ The advantage of the MNC algorithm over standard parallel tempering lies in the fact that we can choose any path in the field-temperature plane. Although parallel tempering can also be implemented with a variable field, the method does not perform efficiently when systems have disorder $\underline{28}$

In the MNC algorithm 21 a cluster of connected spins is grown between two replicas with the same disorder but at different points in the parameter space, i.e., $(T, B)$ and $\left(T^{\prime}, B^{\prime}\right)$, where $T$ represents the temperature and $B$ the external field (here for the case of the DAFF). Starting from an arbitrary spin with different sign in both realizations, adjacent spins pointing in the same direction are successively added to the cluster with probability

$$
p\left(\beta, \beta^{\prime}\right)=1-\exp \left\{-2\left(\beta+\beta^{\prime}\right)\right\},
$$

where $\beta=1 / T$ is the inverse temperature. Once no more spins can be added to the cluster $\mathcal{C}$, it flips with the Metropolis probability ${ }^{29} \min \{1, \exp (-\Sigma)\}$, where

$$
\Sigma_{\mathrm{DAFF}}=2 \operatorname{sign}(\mathcal{C})\left[\left(\beta-\beta^{\prime}\right)\left(n_{++}-n_{--}\right)+\left(B-B^{\prime}\right)|\mathcal{C}|\right]
$$

for the DAFF, and for the RFIM

$\Sigma_{\mathrm{RFIM}}=2 \operatorname{sign}(\mathcal{C})\left[\left(\beta-\beta^{\prime}\right)\left(n_{++}-n_{--}\right)-\left(h-h^{\prime}\right) \sum_{i \in \mathcal{C}} \delta_{i}\right]$.

Here $|\mathcal{C}|$ is the number of spins in the cluster, $\operatorname{sign}(\mathcal{C})$ the orientation of the spin in the replica having inverse temperature $\beta, n_{++}$and $n_{--}$are the number of bonds connecting to nearest neighbors of the cluster with spin up and spin down in both replicas, respectively. After each cluster update, $(L / 2)^{3}$ attempts to flip single spins are performed, where $L$ is the linear size of the system.
As stated before, the MNC algorithm enables us to perform simulations along any arbitrary path in parameter space. We denote such path a replica chain (RC). The phase boundaries for the RFIM and DAFF in the field-temperature plane are well described by ellipses (see below). To reduce corrections to finite-size scaling 30.31 we therefore choose paths in the field-temperature plane that cut the phase boundaries at as orthogonal an angle as possible. This means that, in general, $T \sim h$ for the RFIM and $T \sim B$ for the DAFF. To ensure efficient mixing and therefore fast convergence of the Monte Carlo method, we additionally connect the point with the highest field within the disordered phase to another RC that runs parallel to the approximated phase boundary to a temperature $T>T_{c}$ and $B=0$ ( $h=0$ for the RFIM), where $T_{c}$ is the critical temperature of the model at zero field (see Fig. 5, light dashed lines). This end point of the second RC is simulated efficiently by the Wolff cluster algorithm. ${ }^{32}$ Simulation parameters are listed in Tables [ and I for the RFIM and DAFF, for the first RCs, respectively.

Finally, we also study the DAFF at zero temperature using the method introduced in Refs. 33 and 34 . Here, the DAFF is mapped onto a graph ${ }^{35}$ with $N$ nodes $(N$ is the number of spins) attached to a source and a sink node, all connected in a distinct manner via edges with positive edge weights. The edge weights are calculated depending on the local staggered field, i.e., $\pm B$. The maximum flow/minimum cut is obtained using the algorithm introduced in Ref. 36. The minimum cut is a direct representation of the ground-state spin configuration from which derived quantities, such as a zerotemperature Binder ratio, can be calculated. Note that the method takes the ground-state degeneracy into account. The simulation parameters for the DAFF at zero temperature are shown in Table III]

\section{OBSERVABLES}

Both the DAFF and RFIM undergo second-order phase transitions as a function of temperature and field. To pinpoint the transition temperature, we measure the Binder cumulant $\frac{37}{3}$ as well as the two-point finite-size correlation function $\underline{38} \underline{\underline{40}}$ To compute these observables, we measure the magnetization per spin

$$
M=\frac{1}{N} \sum_{i}^{N} S_{i} .
$$

For the DAFF we measure the staggered magnetization, i.e., each second spin is counted opposite to its orientation in a three-dimensional checker-board manner. For simplicity, we refer to the staggered magnetization also as $M$. An antiferromagnetically-ordered spin configuration has therefore $M=1$. A Binder cumulant for $M$ can 
TABLE I: Simulation parameters for the RFIM along different nontrivial paths of the type $h=a+b T$ in the $h-T$ plane for different linear system sizes $L$ (the first two path types have $b=0)$ ). $\quad N_{\mathrm{sa}}$ is the number of disorder realizations. $N_{T}$ corresponds to the number of temperatures (points) along the simulation path. $T_{\min }$ and $T_{\max }$ are the lowest and highest temperature simulated, respectively. The equilibration/measurement times are $2^{x}$ Monte Carlo sweeps.

\begin{tabular}{lrrrrrr}
\hline \hline simulation path & $L$ & $N_{\text {sa }}$ & $N_{T}$ & $T_{\min }$ & $T_{\max }$ & $x$ \\
\hline$h=0.225$ & 8 & 1536 & 25 & 4.00 & 5.00 & 18 \\
$h=0.225$ & 10 & 827 & 25 & 4.00 & 5.00 & 18 \\
$h=0.225$ & 12 & 2048 & 17 & 4.30 & 4.80 & 18 \\
$h=0.225$ & 16 & 1024 & 19 & 4.35 & 4.70 & 18 \\
$h=0.225$ & 20 & 1024 & 19 & 4.35 & 4.70 & 18 \\
$h=0.225$ & 24 & 1024 & 26 & 4.40 & 4.69 & 18 \\
$h=0.225$ & 28 & 666 & 26 & 4.40 & 4.69 & 18 \\
$h=0.225$ & 32 & 406 & 26 & 4.40 & 4.69 & 18 \\
$h=0.225$ & 36 & 1017 & 26 & 4.40 & 4.69 & 18 \\
$h=0.5$ & 10 & 2503 & 17 & 4.20 & 4.60 & 18 \\
$h=0.5$ & 12 & 4035 & 17 & 4.20 & 4.60 & 18 \\
$h=0.5$ & 16 & 2048 & 17 & 4.20 & 4.60 & 18 \\
$h=0.5$ & 20 & 1024 & 14 & 4.30 & 4.50 & 18 \\
$h=0.5$ & 512 & 14 & 4.30 & 4.50 & 18 \\
$h=1.22 T-3.43$ & 10 & 4096 & 15 & 3.40 & 4.10 & 18 \\
$h=1.22 T-3.43$ & 12 & 3852 & 15 & 3.40 & 4.10 & 18 \\
$h=1.22 T-3.43$ & 16 & 1177 & 17 & 3.65 & 4.10 & 18 \\
$h=1.22 T-3.43$ & 18 & 862 & 17 & 3.65 & 4.10 & 18 \\
$h=1.22 T-3.43$ & 20 & 957 & 17 & 3.60 & 4.00 & 18 \\
$h=1.22 T-3.43$ & 24 & 976 & 17 & 3.60 & 4.00 & 18 \\
$h=1.22 T-3.43$ & 28 & 646 & 17 & 3.60 & 4.00 & 18 \\
$h=1.22 T-3.43$ & 32 & 379 & 17 & 3.60 & 4.00 & 18 \\
$h=2.67 T-6.10$ & 8 & 4071 & 25 & 2.80 & 3.06 & 18 \\
$h=2.67 T-6.10$ & 10 & 4045 & 25 & 2.80 & 3.06 & 18 \\
$h=2.67 T-6.10$ & 12 & 512 & 27 & 2.85 & 3.00 & 18 \\
$h=2.67 T-6.10$ & 14 & 512 & 27 & 2.85 & 3.00 & 18 \\
$h=2.67 T-6.10$ & 16 & 605 & 17 & 2.85 & 2.95 & 18 \\
$h=2.67 T-6.10$ & 18 & 1024 & 27 & 2.85 & 3.05 & 18 \\
$h=2.67 T-6.10$ & 20 & 512 & 31 & 2.86 & 2.93 & 18 \\
$h=2.67 T-6.10$ & 22 & 981 & 31 & 2.85 & 3.05 & 18 \\
$h=2.67 T-6.10$ & 24 & 1024 & 31 & 2.85 & 3.05 & 18 \\
$h=4.94 T-6.80$ & 16 & 1912 & 15 & 1.76 & 1.88 & 18 \\
$h=4.94 T-6.80$ & 18 & 2048 & 15 & 1.76 & 1.88 & 18 \\
$h=4.94 T-6.80$ & 20 & 1858 & 15 & 1.76 & 1.89 & 18 \\
$h=4.94 T-6.80$ & 24 & 906 & 15 & 1.76 & 1.89 & 18 \\
$h=4.94 T-6.80$ & 28 & 505 & 15 & 1.76 & 1.89 & 18 \\
$h=4.94 T-6.80$ & 32 & 627 & 15 & 1.76 & 1.89 & 18 \\
\hline \hline & & & & & & \\
$h$ & & & & & &
\end{tabular}

then be defined via

$$
g(T, L)=\frac{1}{2}\left(3-\frac{\left[\left\langle M^{4}\right\rangle\right]_{\mathrm{av}}}{\left[\left\langle M^{2}\right\rangle^{2}\right]_{\mathrm{av}}}\right),
$$

where $\langle\cdots\rangle$ represents a thermal average and $[\cdots]_{\text {av }}$ an average over disorder (field or dilution configurations) for a fixed value of $h(\mathrm{RFIM})$ or $B$ (DAFF). Close to criticality the Binder ratio scales as

$$
g(T, L)=\tilde{G}\left[L^{1 / \nu}\left(T-T_{c}\right)\right],
$$

where $\tilde{G}$ is a universal function. Note that for the DAFF, when $T=0, g(B, L)=\tilde{G}^{\prime}\left[L^{1 / \nu}\left(B-B_{c}\right)\right]$. To com-
TABLE II: Simulation parameters for the DAFF along nontrivial paths of the type $B=a+b T$ in the $B-T$ plane for different linear system sizes $L$ (the first two path types have $b=0) . \quad N_{\mathrm{sa}}$ is the number of disorder realizations. $N_{T}$ corresponds to the number of temperatures (points) along the simulation path. $T_{\min }$ and $T_{\max }$ are the smallest and the highest temperatures of the $\mathrm{RC}$, respectively. The equilibra-

\begin{tabular}{|c|c|c|c|c|c|c|}
\hline simulation path & $L$ & $N_{\mathrm{sa}}$ & $N_{T}$ & $T_{\min }$ & $\overline{T_{\max }}$ & $x$ \\
\hline $\bar{B}=0.1$ & 8 & 2166 & 26 & 2.50 & 3.50 & 18 \\
\hline$B=0.1$ & 12 & 1208 & 26 & 2.50 & 3.50 & 18 \\
\hline$B=0.1$ & 14 & 1042 & 18 & 2.70 & 3.30 & 18 \\
\hline$B=0.1$ & 16 & 2048 & 19 & 2.80 & 3.30 & 18 \\
\hline$B=0.1$ & 18 & 1104 & 19 & 2.80 & 3.30 & 18 \\
\hline$B=0.1$ & 20 & 796 & 21 & 2.80 & 3.35 & 18 \\
\hline$B=0.1$ & 24 & 444 & 21 & 2.80 & 3.35 & 18 \\
\hline$B=0.1$ & 28 & 505 & 21 & 2.80 & 3.35 & 18 \\
\hline$B=0.1$ & 32 & 322 & 21 & 2.80 & 3.35 & 18 \\
\hline$B=1.0$ & 14 & 1271 & 21 & 2.70 & 3.20 & 18 \\
\hline$B=1.0$ & 16 & 1718 & 21 & 2.70 & 3.20 & 18 \\
\hline$B=1.0$ & 18 & 1215 & 21 & 2.70 & 3.20 & 18 \\
\hline$B=1.0$ & 20 & 888 & 21 & 2.70 & 3.20 & 18 \\
\hline$B=1.0$ & 24 & 491 & 21 & 2.70 & 3.20 & 18 \\
\hline$B=1.0$ & 28 & 556 & 21 & 2.70 & 3.20 & 18 \\
\hline$B=1.0$ & 32 & 352 & 21 & 2.70 & 3.20 & 18 \\
\hline$B=0.2 T$ & 8 & 1344 & 17 & 2.55 & 3.30 & 18 \\
\hline$B=0.2 T$ & 10 & 685 & 17 & 2.55 & 3.30 & 18 \\
\hline$B=0.2 T$ & 12 & 452 & 17 & 2.55 & 3.30 & 18 \\
\hline$B=0.2 T$ & 16 & 542 & 31 & 2.87 & 3.50 & 18 \\
\hline$B=0.2 T$ & 20 & 1564 & 31 & 2.87 & 3.50 & 18 \\
\hline$B=0.2 T$ & 22 & 825 & 31 & 2.87 & 3.50 & 18 \\
\hline$B=0.2 T$ & 24 & 189 & 31 & 2.87 & 3.50 & 18 \\
\hline$B=0.2 T$ & 26 & 128 & 31 & 2.87 & 3.50 & 18 \\
\hline$B=0.2 T$ & 28 & 115 & 31 & 2.87 & 3.50 & 18 \\
\hline$B=0.2 T$ & 30 & 558 & 31 & 2.87 & 3.50 & 18 \\
\hline$B=0.2 T$ & 32 & 383 & 31 & 2.87 & 3.50 & 18 \\
\hline$B=0.67 T$ & 10 & 1201 & 30 & 2.45 & 3.50 & 18 \\
\hline$B=0.67 T$ & 12 & 711 & 30 & 2.45 & 3.50 & 18 \\
\hline$B=0.67 T$ & 16 & 305 & 30 & 2.45 & 3.50 & 18 \\
\hline$B=0.67 T$ & 20 & 512 & 27 & 2.35 & 3.50 & 18 \\
\hline$B=0.67 T$ & 22 & 1024 & 27 & 2.35 & 3.50 & 18 \\
\hline$B=0.67 T$ & 24 & 2048 & 30 & 2.35 & 3.50 & 18 \\
\hline$B=0.67 T$ & 28 & 1024 & 27 & 2.35 & 3.50 & 18 \\
\hline$B=0.67 T$ & 32 & 741 & 30 & 2.37 & 3.50 & 18 \\
\hline$B=1.5 T$ & 10 & 1920 & 17 & 1.30 & 1.62 & 18 \\
\hline$B=1.5 T$ & 12 & 1984 & 17 & 1.30 & 1.62 & 18 \\
\hline$B=1.5 T$ & 16 & 2048 & 17 & 1.30 & 1.62 & 18 \\
\hline$B=1.5 T$ & 18 & 2048 & 26 & 1.30 & 3.50 & 18 \\
\hline$B=1.5 T$ & 20 & 1056 & 20 & 1.35 & 1.60 & 18 \\
\hline$B=1.5 T$ & 24 & 807 & 20 & 1.35 & 1.60 & 18 \\
\hline$B=1.5 T$ & 28 & 457 & 20 & 1.35 & 1.60 & 18 \\
\hline$B=1.5 T$ & 32 & 532 & 20 & 1.35 & 1.60 & 18 \\
\hline$B=1.5 T$ & 36 & 336 & 20 & 1.35 & 1.60 & 18 \\
\hline
\end{tabular}
tion/measurement times are $2^{x}$ Monte Carlo sweeps.

pute the two-point finite-size correlation function we first calculate the wave-vector-dependent susceptibility (along 
TABLE III: Simulation parameters for the DAFF at zero temperature for different fields $B$ and for different linear system sizes $L . N_{\mathrm{sa}}$ is the number of disorder realizations. $B_{\min }$ and $B_{\max }$ are the lowest and highest fields simulated, and $N_{B}$ corresponds to the number of fields simulated to perform a finite-size scaling analysis.

\begin{tabular}{lrccc}
\hline \hline$L$ & $N_{\text {sa }}$ & $B_{\min }$ & $B_{\max }$ & $N_{B}$ \\
\hline 24 & 10302 & 2.00 & 4.30 & 31 \\
32 & 2091 & 2.40 & 2.70 & 16 \\
48 & 2091 & 2.10 & 2.80 & 17 \\
64 & 2091 & 2.30 & 2.70 & 21 \\
72 & 2040 & 2.30 & 2.54 & 17 \\
96 & 5100 & 2.30 & 2.54 & 17 \\
128 & 3586 & 2.30 & 2.47 & 22 \\
\hline \hline
\end{tabular}

the $x$ direction) via

$$
\chi(\mathbf{k})=\left[\left\langle\left(\frac{1}{N} \sum_{j} S_{j} e^{i k x_{j}}\right)^{2}\right\rangle\right]_{\mathrm{av}} .
$$

The two-point finite-size correlation function is then given by

$$
\xi_{L}=\frac{1}{2 \sin \left(k_{\min } / 2\right)} \sqrt{\frac{\chi(\mathbf{0})}{\chi\left(\mathbf{k}_{\min }\right)}-1}
$$

with $\mathbf{k}_{\min }=(2 \pi / L, 0,0)$. The two-point finite-size correlation function scales as

$$
\xi_{L}(T, L) / L=\tilde{X}\left[L^{1 / \nu}\left(T-T_{c}\right)\right] .
$$

Using both the Binder ratio and the two-point finitesize correlation function allows us to perform a detailed finite-size scaling analysis to determine the critical exponent $\nu$, as well as to test if both models share the same universality class using the method introduced in Ref. 41. To obtain an optimal data collapse we use a Levenberg-Marquardt minimization combined with a bootstrap analysis, see Ref. 41. This allows us to determine the optimal values of the critical parameters $T_{c}$ and $\nu$ with a statistical error bar by fitting the data to a thirdorder polynomial that approximates the scaling functions $\tilde{G}(x)$ and $\tilde{X}(x)$ close to $x=0$, where $x=L^{1 / \nu}\left(T-T_{c}\right)$.

Finally, to determine the critical exponent $\eta$, we determine the peak position of the connected susceptibility given by

$$
\chi=\frac{1}{T}\left(\left[\left\langle M^{2}\right\rangle\right]_{\mathrm{av}}-[\langle M\rangle]_{\mathrm{av}}^{2}\right)
$$

where the magnetization $M$ is given by Eq. (6). Note that the connected susceptibility is related to Eq. (9) in the limit of zero wave vector. Furthermore, in the thermodynamic limit $[\langle M\rangle]_{\mathrm{av}}=0$ for $T=T_{c}$ so, in principle, Eq. (9) could also be used for the analysis. In general, the susceptibility scales as

$$
\chi \sim L^{2-\eta} \widetilde{C}\left[L^{1 / \nu}\left(T-T_{c}\right)\right] .
$$

Therefore, when $T=T_{c}$ the function $\widetilde{C}$ is a constant independent of the system size and $\chi \sim L^{2-\eta}$ from which the exponent $\eta$ can be determined.

\section{RESULTS}

The critical parameters for both the RFIM and the DAFF have been computed via a finite-size scaling analysis of the two-point finite-size correlation function [Eq. (11)] along the different simulation paths. Finitesize corrections can be large for small system sizes and are strongly field dependent, which is why for some external fields in both models we do not include small systems in the finite-size scaling analysis used to determine the critical parameters. To illustrate the typical behavior, in Fig. 1] left panel, we show the two-point finite-size correlation function for the DAFF for $B=1.0$ and different system sizes. The data cross at a point, therefore signaling the existence of a phase transition. Note that for this particular field corrections to scaling are manageable and the data scale well, as can be seen in Fig. 1, right panel. However, this is not always the case, especially when the external field is large. For the RFIM corrections to scaling are considerably stronger, even at small fields, see Fig. 2,

Using finite-size scaling we determine the location of the critical points, as well as the associated critical exponent $\nu$ for the different simulation paths. In addition, we also compute the critical exponent $\eta$ by studying the finite-size behavior of the susceptibility peak. Data for the RFIM are summarized in Table IV for the DAFF in Table V

TABLE IV: Critical temperature $T_{c}$ and critical field $h_{c}$ computed from a finite-size scaling analysis of the two-point finitesize correlation function for the RFIM. $\nu$ is the critical exponent of the correlation length. The exponent $\eta$ is computed from the peak of the susceptibility.

\begin{tabular}{lllll}
\hline \hline simulation path & $T_{c}$ & $h_{c}$ & $\nu$ & $\eta$ \\
\hline$h=0.225$ & $4.481(1)$ & 0.225 & $1.39(4)$ & $0.082(1)$ \\
$h=0.5$ & $4.381(2)$ & 0.5 & $1.30(5)$ & $0.202(16)$ \\
$h=1.22 T-3.4$ & $3.76(2)$ & $1.16(3)$ & $1.39(5)$ & $0.92(40)$ \\
$h=2.70 T-6.1$ & $2.89(5)$ & $1.7(1)$ & $1.3(1)$ & $0.47(15)$ \\
$h=4.94 T-6.8$ & $1.79(1)$ & $2.01(5)$ & $1.4(1)$ & $0.85(4)$ \\
\hline \hline
\end{tabular}

To determine the critical field $B_{c}$ at zero temperature for the DAFF we compute ground states with the algorithm introduced in Ref. 34. The same finite-size scaling technique as used for the two-point finite-size correlation function (see above) can be used to analyze the ground-state Binder cumulant. The data collapse is shown in Fig. 3. The results for the critical point and the correlation-length exponent at zero temperature are stated in the last line of Table $\mathrm{V}$.

We also determine the peak position of the fluctuations 

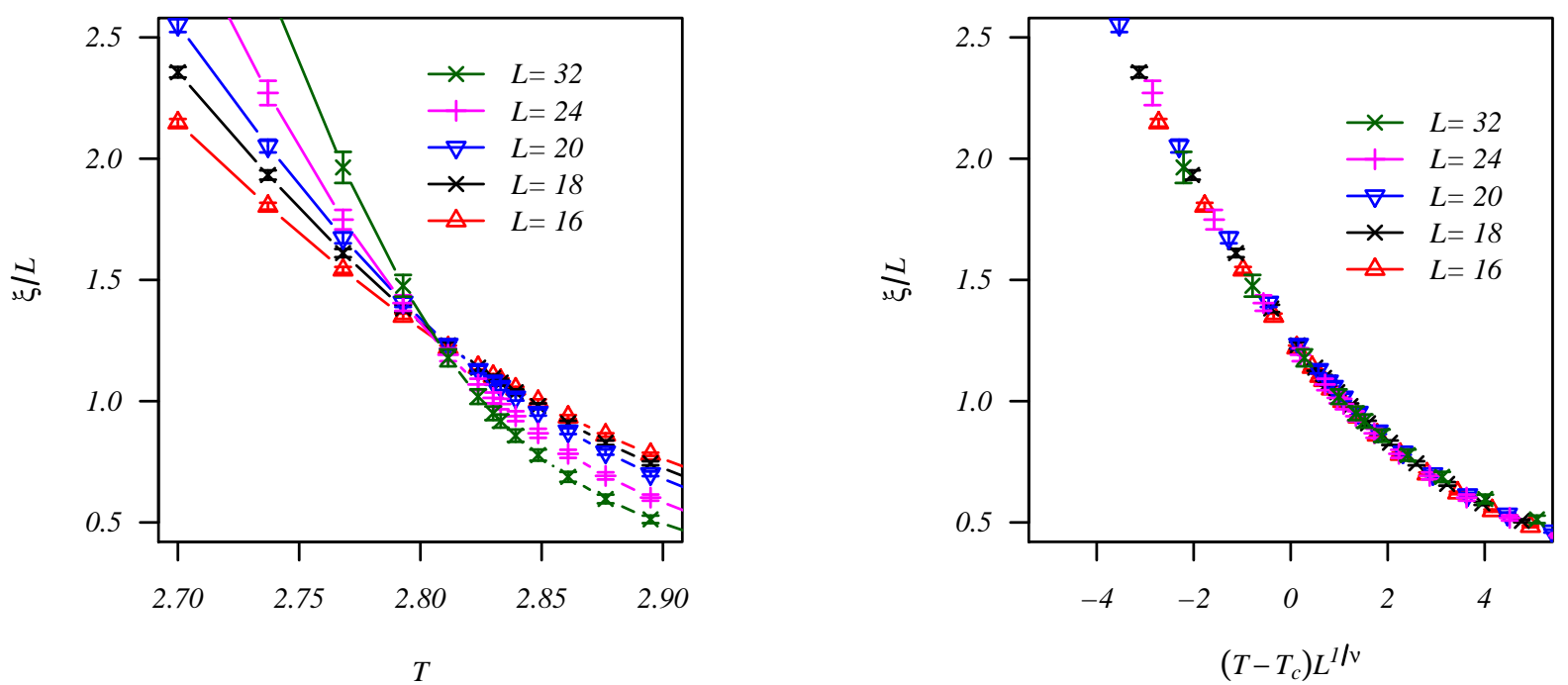

FIG. 1: (Color online) Left: Two-point finite-size correlation function $\xi_{L} / L$ vs temperature $T$ for the DAFF with $B=1.0$ and different linear system sizes $L$. Finite-size corrections are small and the data cross at one point signaling a transition. Right: Finite-size scaling collapse of the data in the left panel. The best collapse is obtained with $T_{c} \approx 2.807$ and $\nu \approx 1.2$.
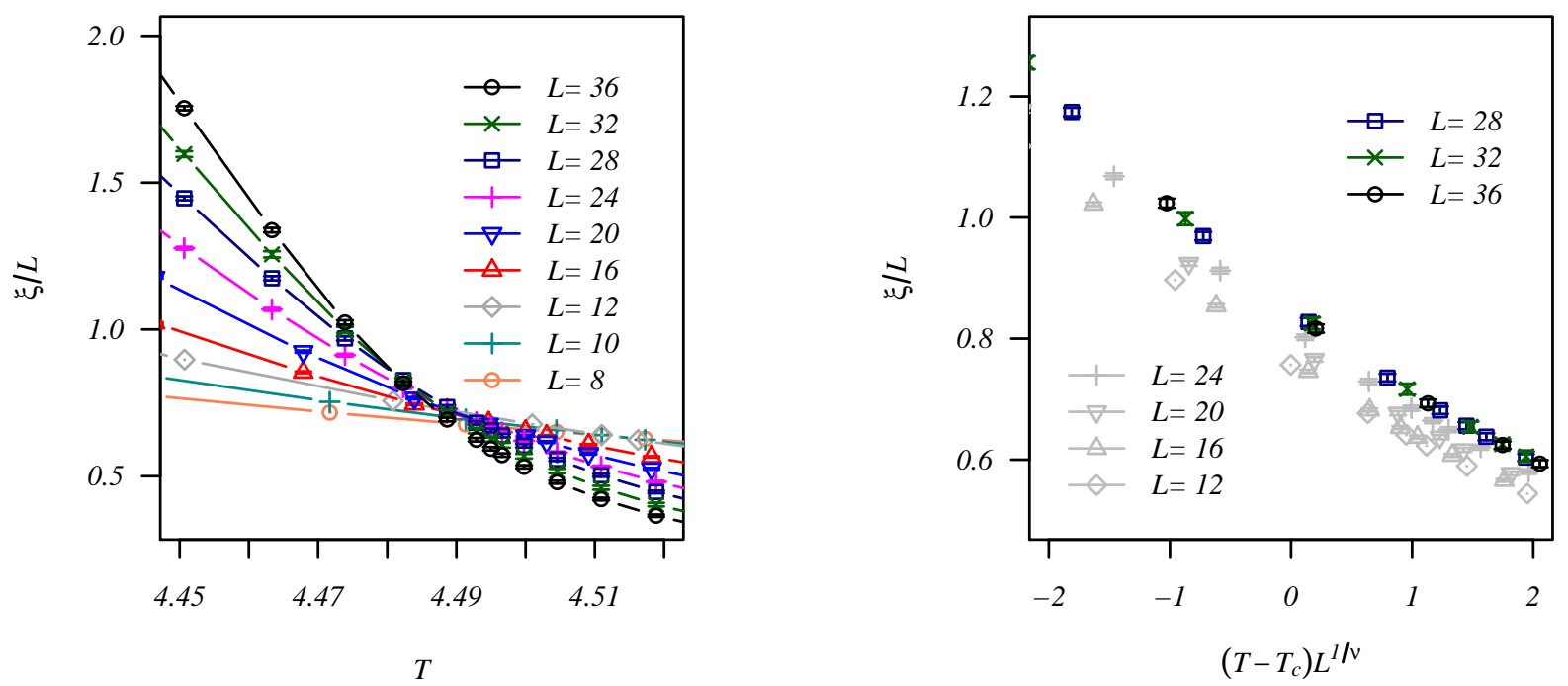

FIG. 2: (Color online) Left: Two-point finite-size correlation function $\xi_{L} / L$ vs temperature $T$ for the RFIM with $h=0.225$ and different linear system sizes $L$. Finite-size corrections are large. Right: Finite-size scaling collapse of the data in the left panel. Because of the large corrections to scaling, only data for $L \geq 28$ are used in the scaling collapse. Data for $L \leq 24$ (light shaded) are not included in the data collapse and shown to illustrate the corrections to scaling. The best collapse is obtained with $T_{c} \approx 4.481$ and $\nu \approx 1.39$.

of the staggered magnetization of the ground states:

$$
\mathcal{F}(B)=L^{3}\left(\left[M^{2}\right]_{\text {av }}-[M]_{\text {av }}^{2}\right) .
$$

This approach has proven to be quite accurate in previous studies for the susceptibility $\underline{\underline{47}}$ Because the fluctuations peak at the putative transition, we fit a Gaus- sian to the peak and determine its precise location. Error bars are determined via a configurational bootstrap

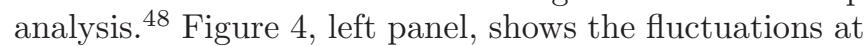
zero temperature and as a function of the applied field $B$. The peaks are well described by Gaussians. The right panel of Fig. (4 shows an extrapolation of the peak 
TABLE V: Critical temperature $T_{c}$ and critical field $B_{c}$ computed from a finite-size scaling analysis of the two-point finitesize correlation function for the DAFF. $\nu$ is the critical exponent of the correlation length. The exponent $\eta$ is computed from the peak of the susceptibility. Note that estimating $\eta$ was not possible for $B=1.5$. The last line lists data from zero-temperature simulations (see text). The estimate of the critical field $B_{c}$ is obtained from a finite-size scaling analysis of the zero-temperature Binder ratio.

\begin{tabular}{lllll}
\hline \hline simulation path & $T_{c}$ & $B_{c}$ & $\nu$ & $\eta$ \\
\hline$B=0.1$ & $2.977(1)$ & 0.1 & $1.34(5)$ & $0.406(26)$ \\
$B=1.0$ & $2.807(1)$ & 1.0 & $1.2(2)$ & $0.023(12)$ \\
$B=0.2 T$ & $2.908(4)$ & $0.582(8)$ & $1.36(7)$ & $0.11(2)$ \\
$B=0.67 T$ & $2.42(1)$ & $1.61(1)$ & $1.5(3)$ & $0.67(5)$ \\
$B=1.5$ & $1.46(9)$ & $2.2(1)$ & $1.4(3)$ & - \\
$T=0$ & 0 & $2.32(2)$ & $1.43(2)$ & $0.68(1)$ \\
\hline \hline
\end{tabular}

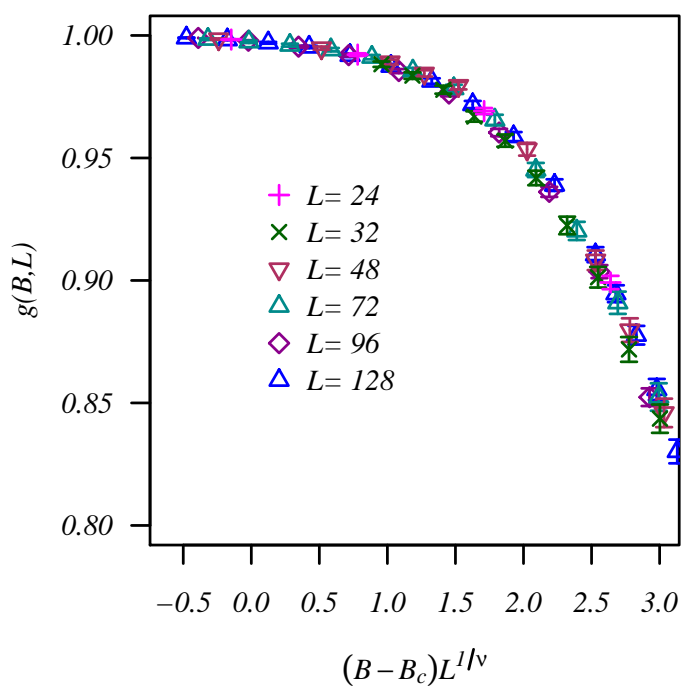

FIG. 3: (Color online) Data collapse of the zero-temperature Binder cumulant of the DAFF as a function of the reduced scaling variable $\left(B-B_{c}\right) L^{1 / \nu}$ for different system sizes. The best collapse is obtained for $B_{c}^{0} \approx 2.32$ and $\nu \approx 1.43$.

position to infinite system size assuming the functional form $B_{c}(L)=B_{c}+a L^{-\omega}$. The best fit is obtained for $B_{c}=2.34(2)[\omega=1.25(9)]$, in agreement with the estimate using the Binder cumulant, see Table $\nabla$

Combining the data in Table $\mathbf{V}$ with some values from the literature $\underline{\underline{42}-\underline{44}, 49}$ we can approximate to good accuracy the phase boundary for the DAFF via

$$
\left(\frac{B_{c}}{\tilde{B}_{c}^{0}}\right)^{1.81}+\left(\frac{T_{c}}{\tilde{T}_{c}^{0}}\right)^{3.54}=1
$$

with $\tilde{T}_{c}^{0} \approx 2.980$ and $\tilde{B}_{c}^{0} \approx 2.31$. Similarly, using the data from Table IV and known values from the literature $45,46,50$ we obtain for the RFIM

$$
\left(\frac{h_{c}}{h_{c}^{0}}\right)^{1.95}+\left(\frac{T_{c}}{T_{c}^{0}}\right)^{1.80}=1
$$

with $h_{c}^{0}=2.27 \underline{46}$ and $T_{c}^{0}=4.5115 \underline{\underline{45}}$ Note that the critical phase boundary points $T_{c}^{0}$ and $h_{c}^{0}$ have been determined to high precision in the literature; see Refs. 45 and 46, respectively. Furthermore, for the RFIM with bimodal disorder, a similar elliptical phase diagram has been proposed in Ref. 51. For the DAFF, $\tilde{T}_{c}^{0}$ and $\tilde{B}_{c}^{0}$ are approximated but agree with the numerical estimates we present. In Fig. 5 we show the phase boundaries for the DAFF (left panel) and the RFIM (right panel), together with the simulated critical points. The dashed lines represent the simulation paths taken.

\section{DISCUSSION}

Cardy $\stackrel{17}{ }$ predicted an equivalence between the DAFF and the RFIM for small applied fields using a mean-field argument. This equivalence is often quoted in experimental studies where materials which are diluted antiferromagnets in a field are then described using the RFIM (see, for example, Refs. 3, 12, 15).

Equation (15) in Ref. 17 maps the RFIM onto the DAFF:

$$
h(T)=\frac{p(1-p)\left(T_{c}^{\text {pure }} / T\right)^{2}(B / T)^{2}}{\left(1-\theta^{M F} / T\right)^{2}} .
$$

Here, $p=0.7, T_{c}^{\text {pure }}=4.5115$, and $\theta^{M F}=2 d J=6$ is the mean-field coupling strength. We can now use the obtained phase boundaries [Eqs. (15) and (16)] to compare both models. Figure 6 shows the phase boundary for the RFIM (solid line, the circles represent the obtained critical points along the different simulation paths) together with the phase boundary for the DAFF mapped onto the RFIM space using Eq. (17) (dashed line, the squares represent the obtained critical points along the different simulation paths for the DAFF). For random-field strengths of up to $h \approx 1.2$ - which means field strengths of up to $B \approx 1.6$ for the DAFF - there is an approximate correspondence between both models. However, as the figure clearly illustrates, strictly speaking the correspondence only seems to work in the limit of $h \rightarrow 0(h \lesssim 0.3)$. Given the mean-field nature of the Cardy argument, the agreement of the phase boundaries is rather good. On the other hand, it is not surprising that for larger disorder, they do not agree exactly. It is of importance to take these limitations of the Cardy mapping 17 into account when studying diluted antiferromagnets in an external field experimentally while attempting to describe the data analytically using the RFIM. Furthermore, a basic finite-size scaling analysis leads to no systematic deviations of the correlation-length exponent $\nu$. Including the estimates for rough simulations at high fields, our 

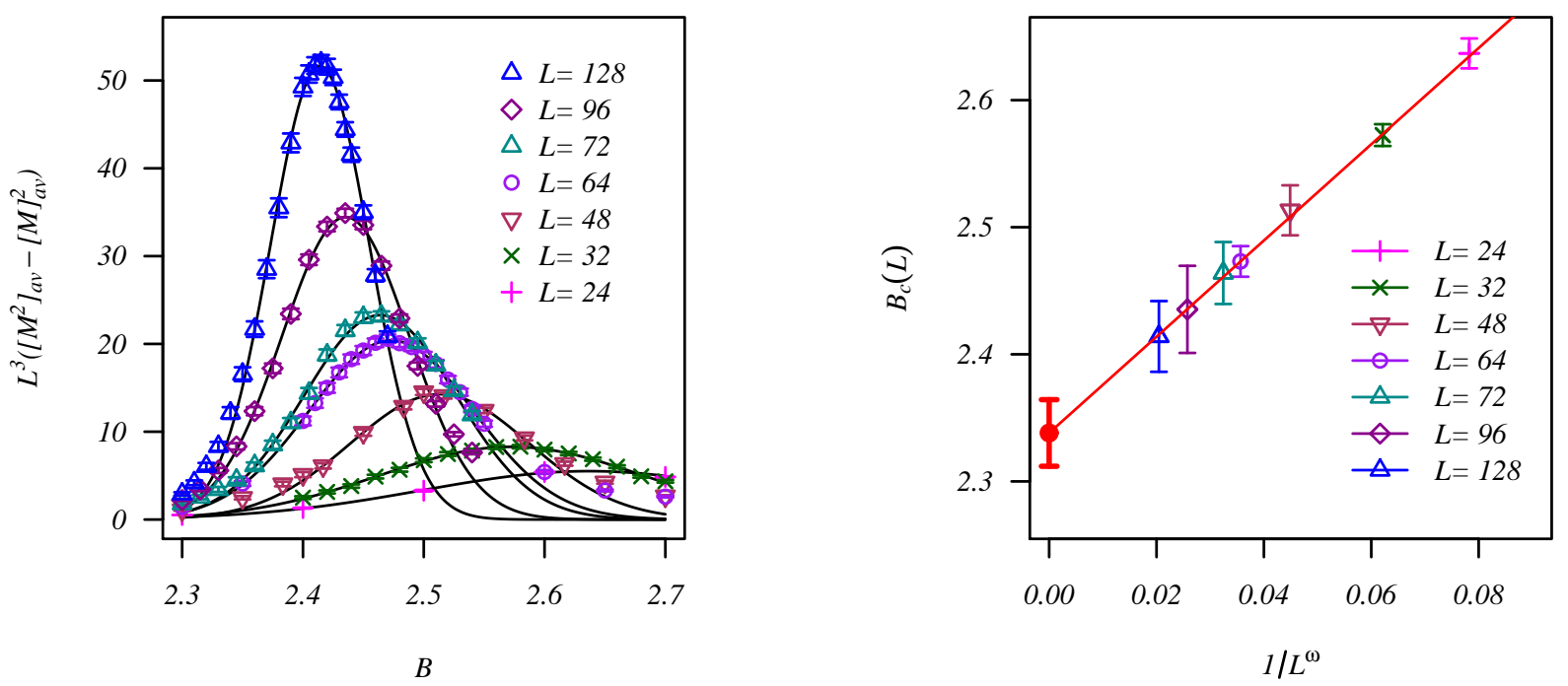

FIG. 4: (Color online) Left: Fluctuations of the staggered magnetization of the DAFF as a function of applied field $B$ for different system sizes. The peak positions signals the presence of a transition. The data are well described by a Gaussian close to the peak (solid lines). To determine the thermodynamic critical field $B_{c}$ we extrapolate the data to infinite system size (right panel) using $B_{c}(L)=B_{c}+a L^{-\omega}$. The best fit is obtained for $B_{c}=2.34(2)$ and $\omega=1.25(9)$. The red (filled) point represents the thermodynamic extrapolation, $B_{c}=2.34(2)$.

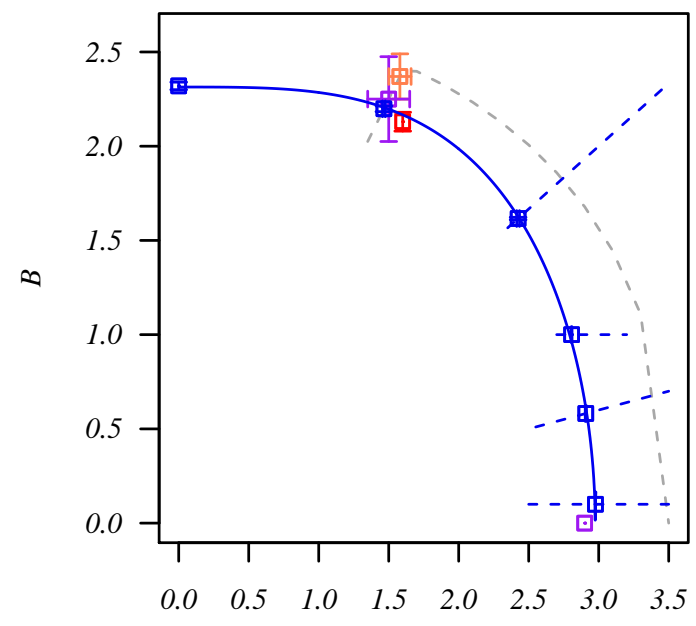

$T$

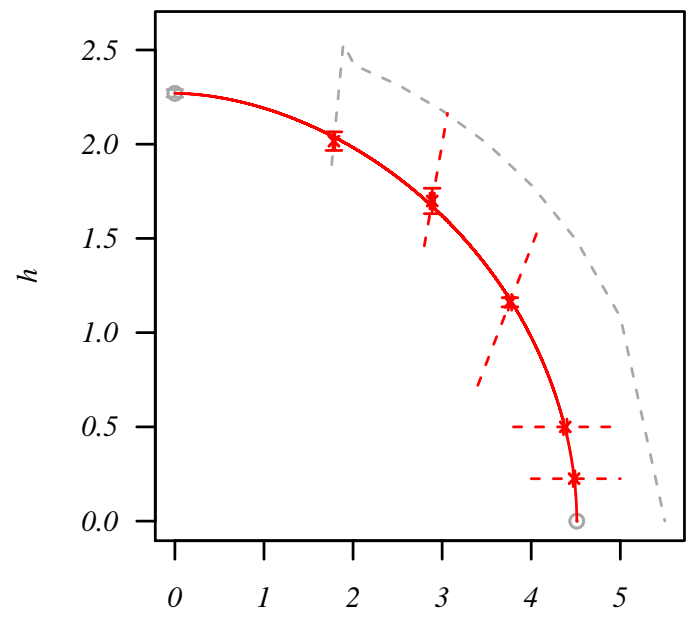

$T$

FIG. 5: (Color online) Left: Empirical phase boundary of the DAFF $(p=0.7)$. The red point is from Ref. 42, the coral point from Ref. 43, and the purple points from Ref. 44. Our data agree within error bars with these previous studies. The blue (solid) curve is given by Eq. (15). The dashed lines represent the parts of the simulation paths that cross the phase boundary. The light shaded line is an example of the second RC that runs parallel to the approximated phase boundary to a temperature $T>T_{c}$ and $B=0$ to speed up equilibration. Right: Empirical phase boundary of the RFIM. The zero-field critical temperature is $T_{c}^{0}=4.5115^{45}$ and $h_{c}^{0}=2.270^{46}$ (gray open circles). The red (solid) curve is given by Eq. (16). The dashed lines represent the parts of the simulation paths that cross the phase boundary. Again, the light shaded line shows an example of the second RC that runs parallel to the approximated phase boundary to a temperature $T>T_{c}$ and $h=0$ to speed up equilibration. 


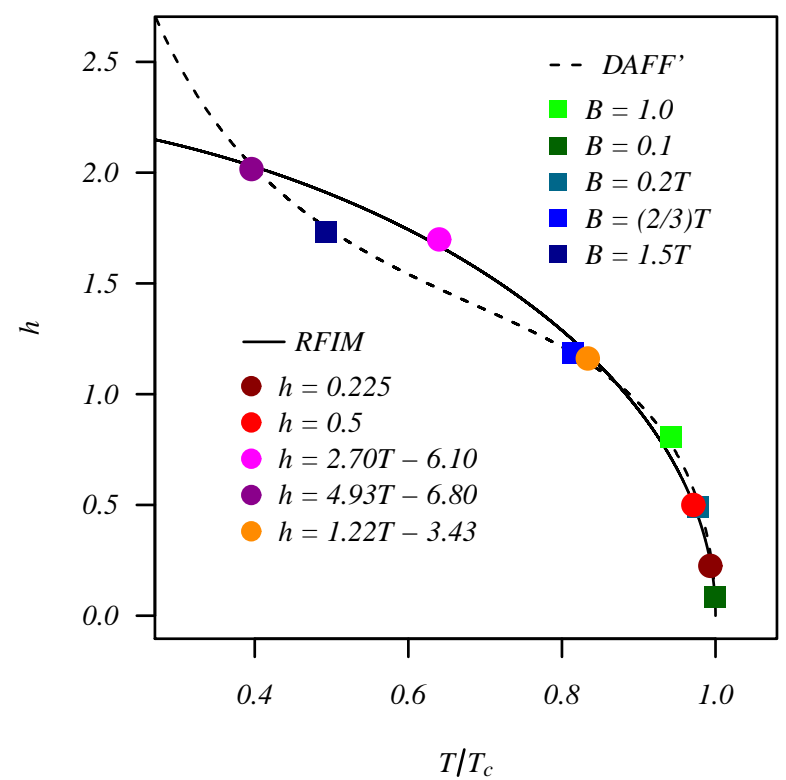

FIG. 6: (Color online) Phase boundary of the RFIM (solid line, from Fig. 5 right panel). The temperature axis has been normalized with $T_{c}(h=0)=4.5115$. The circles represent the different estimates of the critical points along the different simulation paths. The dashed line is the phase boundary computed by applying Eq. (17) to the data of the DAFF. Squares represent the different critical points simulated for the DAFF along the different simulation paths. An approximate correspondence between the phase boundaries only works for fields $h \lesssim 1.2(B \lesssim 1.6$ for the DAFF $)$.

results support

$$
\nu=1.39(17)
$$

for the range of fields studied, in agreement with previous studies, such as $\nu_{\text {RFIM }}=1.37(9) \stackrel{46}{\stackrel{46}{\underline{n}}} \nu=1.20(5) \underline{14}$ from experiments on $\mathrm{Fe}_{0.85} \mathrm{Zn}_{0.15} \mathrm{~F}_{2}(p=0.85)$, or $\nu=$ 1.40 (6) from the disconnected part of the susceptibility of $\mathrm{Fe}_{0.93} \mathrm{Zn}_{0.07} \mathrm{~F}_{2}(p=0.93) .52$ Note that our results are also compatible with the value $\nu=1.10(15)$ computed by Fernandez et al. 42 obtained for their largest system size using the quotient method. They do find other values of $\nu$ for smaller system sizes. Our results are summarized in Fig. 7 As can be clearly seen, the difference between the estimates for the critical exponent of the correlation length for both models is marginal and within error bars: The average estimate for the RFIM is $\bar{\nu}_{\mathrm{RFIM}}=1.37(12)$ (red line in Fig. 7), whereas for the $\mathrm{DAFF} \bar{\nu}_{\mathrm{DAFF}}=1.41(15)$ (blue line in Fig. 7). This apparent agreement of the critical exponent is quite good, given that the proposed equivalence is based on a meanfield argument that typically leads to quite different exponents compared to the true non-mean-field values.

However, the error bars are large and therefore a more detailed study needs to be performed. To truly discern

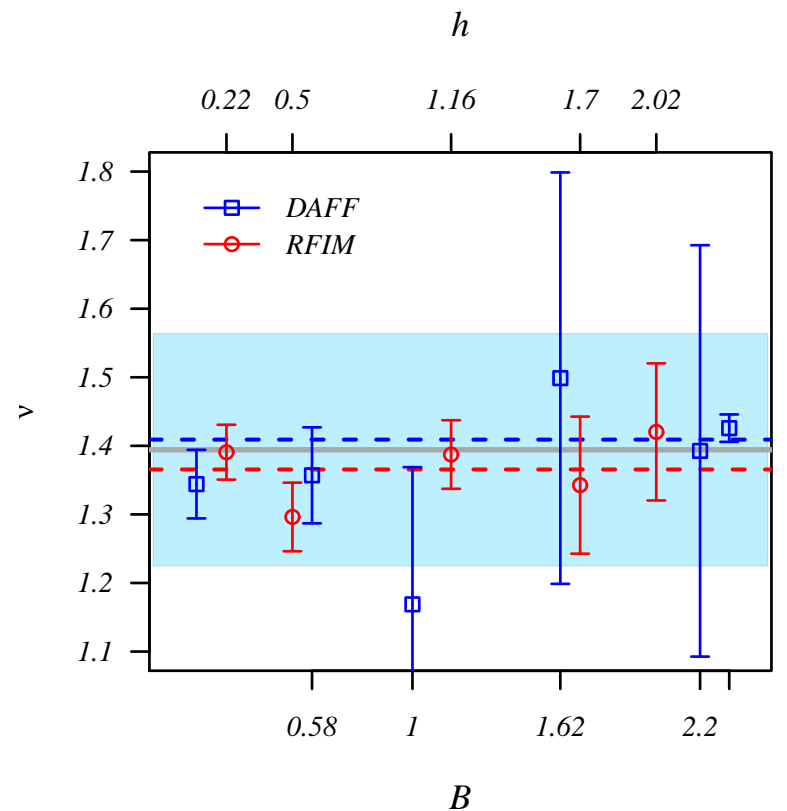

FIG. 7: (Color online) Critical exponent $\nu$ as a function of the field $h$ (RFIM) and $B$ (DAFF). The labels on the upper axis correspond to the random-field strength $h$ (RFIM), those on the lower axis to the external field $B$ (DAFF). The weighted mean is $\nu=1.39(17)$ (gray line) and the weighted error is represented by the shaded (light blue) area. The difference between $\bar{\nu}_{\text {DAFF }}=1.41(15)$ (blue dashed line) and $\bar{\nu}_{\text {RFIM }}=$ $1.37(12)$ (red dashed line) is marginal in comparison to the error-bars of the data points. The RFIM ground-state value is taken from Ref. 46 .

if both models are in the same universality class, in addition to having one (apparently) agreeing critical exponent, one would have to compute a second critical exponent. We also analyzed the behavior of the magnetic susceptibility $\chi$ which has a peak at the phase transition. By studying the finite-size behavior of the peak height (not shown), we determine the critical exponent $\eta$ using the finite-size scaling form of the susceptibility, Eq. (13). Our estimates of the critical exponent $\eta$ along the phase boundary are shown in Fig. 8 and summarized in Tables IV and $\mathrm{V}$ for the RFIM and DAFF, respectively. Fluctuations are very large, especially for large fields, but suggest that both the RFIM and the DAFF might not share the same universality class. For the DAFF, a clear systematic trend is visible that shows that $\eta$ might be strongly field dependent for $B \gtrsim 1.6$, i.e., in the curved portion of the phase boundary. However, note that the exponent $\eta$ is very difficult to compute, as recently shown in Ref. 20. A different approach is the computation of the critical exponent $\alpha$ that describes the divergence of the specific heat. However, for both the RFIM and the DAFF $\alpha$ is close to zero $\stackrel{34,46}{=}$ Therefore, simulations of very large system sizes that are currently not accessible numerically are required. 


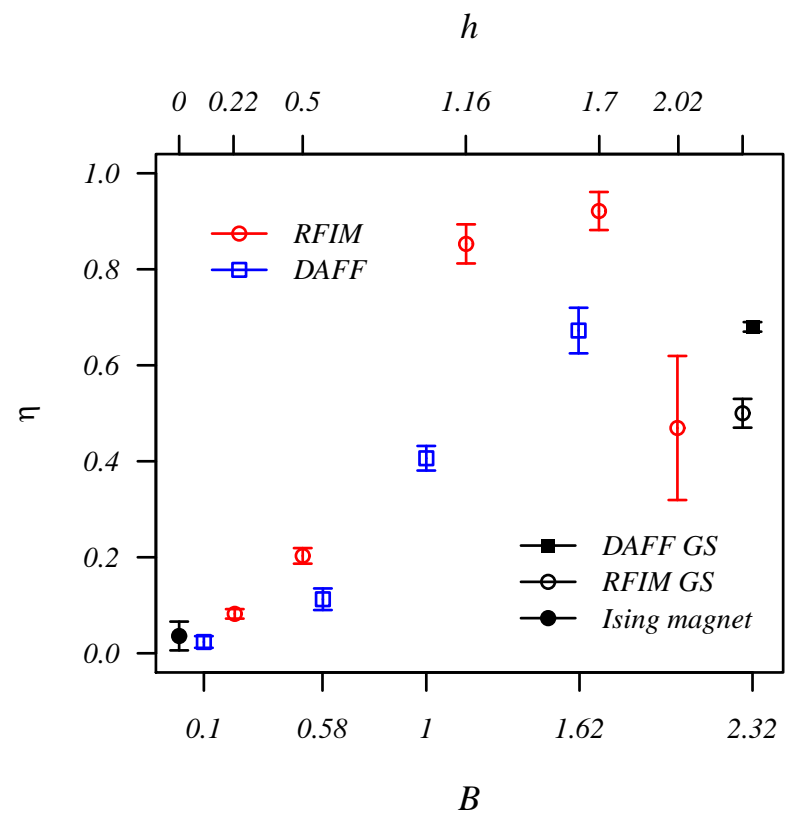

FIG. 8: (Color online) Critical exponent $\eta$ as a function of the field $h$ (RFIM) and $B$ (DAFF). The labels on the upper axis correspond to the random-field strength $h$ (RFIM), those on the lower axis to the external field $B$ (DAFF). For comparison, we also add the estimates for the three-dimensional Ising ferromagnet (filled circle at $h=0$, marked with 'Ising magnet') $\stackrel{45}{\stackrel{4}{5}}$ the RFIM at $T=0$ (open circle at $h=h_{c}$, marked with 'RFIM GS'), ${ }^{50}$ and the DAFF at $T=0$ and $B=B_{c}$ computed from our ground-state data $[\eta(T=0) \approx 0.68(1)$, filled square, marked with 'DAFF GS']. Note that we find very large fluctuations, i.e., a detailed determination of the different universality classes is difficult.

Fortunately, there is a simple yet more sensitive method to verify if two different systems share the same universality class without having to compute any critical exponents: $: 1,53$ Both the Binder cumulant and the two-point finite-size correlation function divided by the system size are dimensionless quantities. By plotting one as a function of the other, nonuniversal quantities cancel

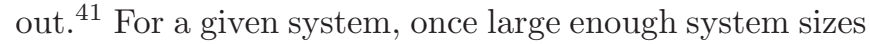
are reached such that corrections to scaling are negligible, the data for all system sizes collapse onto a universal curve within error bars. If two systems share the same critical exponent $\nu$, we expect that all data should collapse onto the same universal curve within error bars and, in particular, that the estimates of the Binder cumulant and the two-point finite-size correlation function agree at the putative critical point(s). We therefore would expect that data sets of $g\left(\xi_{L} / L\right)$ for both the DAFF and the RFIM should agree for all simulated temperatures and, in particular, for $T=T_{c}$.

Figure 9 shows the Binder cumulant as a function of the two-point finite-size correlation function divided by the system size for both the DAFF and the RFIM. The left set of points (reddish/light tones, circles) are for the RFIM. Data for the different simulation paths used collapse onto a master curve. The right set of points (greenish/dark tones, squares) are for the DAFF. Again, all data collapse onto a master curve for all simulation paths taken. This shows that for this type of analysis the finite-size corrections are small for both models and within the statistical fluctuations. However, the data sets for the RFIM and the DAFF do not agree, except in the trivial limit where $g(T) \rightarrow 1$. The large circles for the RFIM (squares for the DAFF) represent our estimates of $g\left(\xi_{L} / L\right)$ at $T=T_{c}$. As can be seen, the data for both models do not agree (i.e., a large circle should sit on top of a large square), something which is even more clear when zooming into the boxed area (inset). Note that the large error bars are due to the uncertainty of the critical temperature. This discrepancy reveals the differences between the DAFF and the RFIM which could not be detected within the scope of a mean-field calculation.

\section{CONCLUSIONS}

We have performed extensive Monte Carlo simulations of the diluted antiferromagnet in a field at $30 \%$ dilution $(p=0.7)$ and the random-field Ising model. Using these data we show that the phase boundaries for both models are well described by ellipses (see Fig. 5). In addition, using zero-temperature heuristic methods, we compute the zero-temperature critical point for the DAFF with $30 \%$ dilution $(p=0.7)$. We expect that the phase boundary for other dilutions will be similar, albeit with different nonuniversal parameters.

Furthermore, we numerically study the equivalence of the RFIM and the DAFF as predicted by Cardy $\underline{17}$ Our results show that only in the limit of small fields do both phase boundaries map onto each other.

Finally, we perform a finite-size scaling analysis to determine the critical exponent $\nu$ of the correlation length. Our results from the two-point finite-size correlation function suggest that the exponent $\nu$ agrees within error bars for both the RFIM and the DAFF. However, error bars are large. To circumvent this problem, we study the Binder cumulant as a function of the two-point finite-size correlation function divided by the system size and show that both models apparently do not share the same universality class. A computation of the exponent $\eta$ is extremely difficult and plagued by finite-size effects. Clearly, more detailed simulations need to be performed to fully discern the critical behavior of both models and fully determine their universality classes. It would be interesting to also measure the critical behavior of the specific heat (critical exponent $\alpha$ ). However, because the exponent is close to zero for both models, large system sizes are needed; sizes that are currently not accessible via simulations. We conclude by cautioning researchers when using the equivalence of both models. 


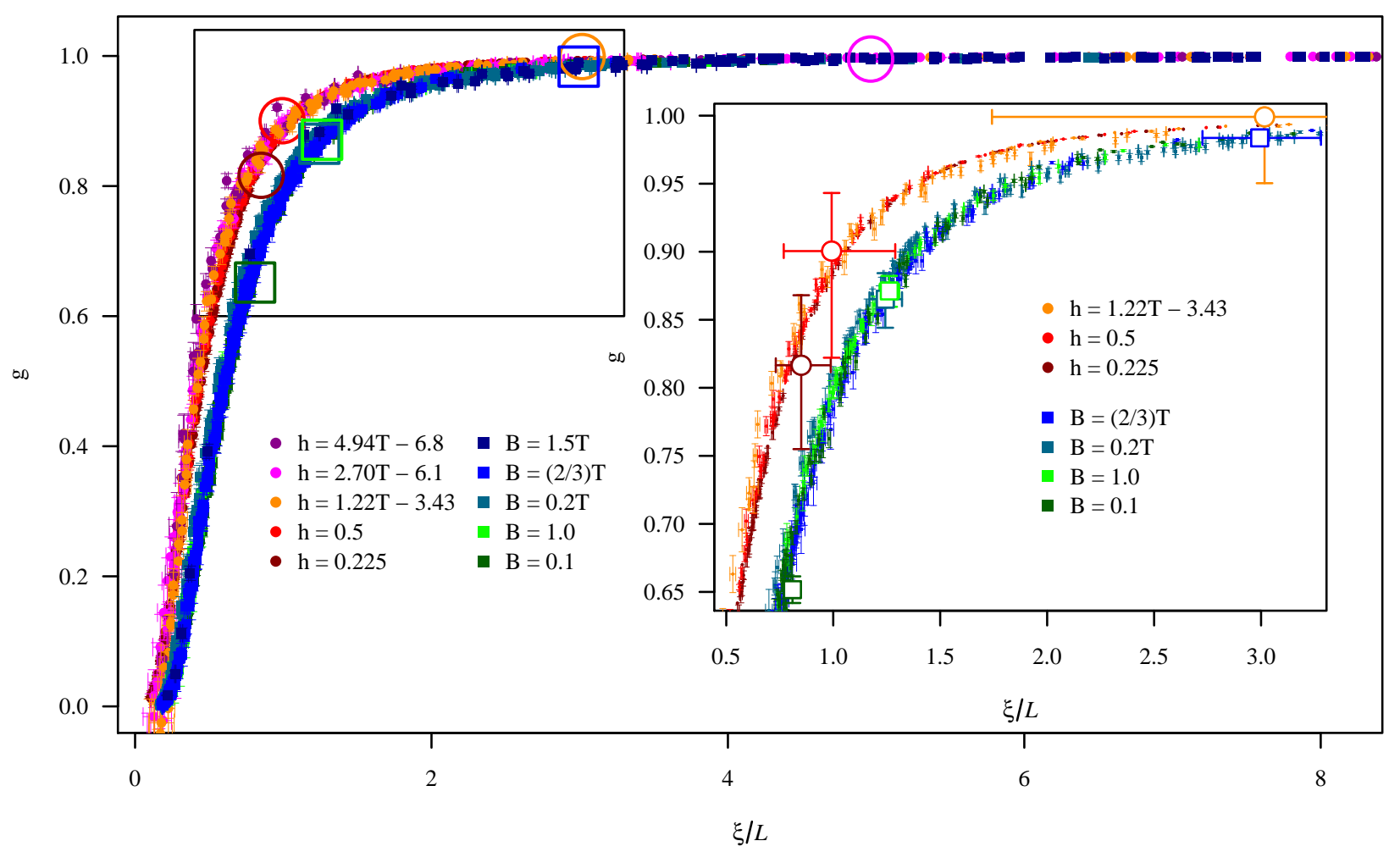

FIG. 9: (Color online) Binder ratio $g$ as a function of the two-point finite-size correlation function divided by the system size $\xi_{L} / L$ for several system sizes and simulation paths. Note that also small system sizes are included, i.e., corrections to finite-size scaling are small. The data for the RFIM and DAFF collapse onto two distinct set of curves, suggesting that both models do not share the same universality class. The left set of points (reddish/light tones, circles) are for the RFIM. The right set of points (greenish/dark tones, squares) are for the DAFF. The large circles for the RFIM (large squares for the DAFF) represent our estimates of $g\left(\xi_{L} / L\right)$ at $T=T_{c}$. The inset zooms into the important region (large box), where the Cardy mapping should apply. Clearly, both data sets are different, suggesting that the RFIM and the DAFF do not share the same universality class.

\section{Acknowledgments}

We would like to thank D. P. Belanger, M. Niemann, and A. P. Young for the fruitful discussions. H.G.K. acknowledges support from the SNF (Grant No. PP002114713) and the NSF (Grant No. DMR-1151387). We would like to thank ETH Zurich for CPU time on the Brutus cluster, Texas A\&M University for CPU time on the Eos cluster, as well as the C. v. O. Universität Oldenburg for CPU time on the Hero cluster funded by the DFG (INST 184/108-1 FUGG) and the ministry of Science and Culture (MWK) of the Lower Saxony State.
1 D. P. Belanger and A. P. Young, The random field Ising model, J. Magn. Magn. Mater. 100, 272 (1991).

2 T. Nattermann, Dipolar interaction in random-field systems, J. Phys. A 21, L645 (1988).

3 D. P. Belanger, Experiments on the Random Field Ising Model, in Spin Glasses and Random Fields, edited by A. P. Young (World Scientific, Singapore, 1998), p. 251.

4 T. Nattermann, Theory of the Random Field Ising Model, in Spin Glasses and Random Fields, edited by A. P. Young (World Scientific, Singapore, 1998), p. 277.

5 I. F. Lyuksyutov, T. Nattermann, and V. Pokrovsky, Theory of the hysteresis loop in ferromagnets, Phys. Rev. B 59, 4260 (1999).
6 A. P. Young, ed., Spin Glasses and Random Fields (World Scientific, Singapore, 1998).

7 J. P. Sethna, K. Dahmen, S. Kartha, J. A. Krumhansl, B. W. Roberts, and J. D. Shore, Hysteresis and hierarchies: Dynamics of disorder-driven first-order phase transformations, Phys. Rev. Lett. 70, 3347 (1993).

8 O. Perkovic, K. A. Dahmen, and J. P. Sethna, Avalanches, Barkhausen Noise, and Plain Old Criticality, Phys. Rev. Lett. 75, 4528 (1995).

${ }^{9}$ O. Perkovic, K. A. Dahmen, and J. P. Sethna, Disorderinduced critical phenomena in hysteresis: Numerical scaling in three and higher dimensions, Phys. Rev. B 59, 6106 (1999). 
10 D. M. Silevitch, G. Aeppli, and T. F. Rosenbaum, Switchable hardening of a ferromagnet at fixed temperature, Proc. Natl. Acad. Sci. U.S.A. 107, 2797 (2010).

11 T. Halpin-Healy, Directed polymers in random media: Probability distributions, Phys. Rev. A 44, R3415 (1991).

${ }^{12} \mathrm{~W}$. Barber and D. Belanger, The random field critical concentration in dilute antiferromagnets, Journal of Applied Physics 87, 7049 (2000).

13 F. Ye, L. Zhou, S. Larochelle, L. Lu, D. P. Belanger, M. Greven, and D. Lederman, Order Parameter Criticality of the $d=3$ Random-Field Ising Antiferromagnet $F e_{0.85} Z n_{0.15} F_{2}$, Phys. Rev. Lett 89, 157202 (2002).

14 F. Ye, M. Matsuda, S. Katano, H. Yoshizawa, D. P. Belanger, E. T. Seppälä, J. A. Fernandez-Baca, and M. J. Alava, Percolation fractal dimension in scattering line shapes of the random-field Ising model, JMMM 272, 1298 (2004).

15 F. Ye, L. Zhou, S. A. Meyer, L. J. Shelton, D. P. Belanger, L. Lu, S. Larochelle, and M. Greven, Quasistationary criticality of the order parameter of the three-dimensional random-field Ising antiferromagnet $\mathrm{Fe}_{0.85} \mathrm{Zn}_{0.15} \mathrm{~F}_{2}$ : A synchrotron x-ray scattering study, Phys. Rev. B 74, 144431 (2006).

16 S. Fishman and A. Aharony, Random field effects in disordered anisotropic antiferromagnets, J. Phys. C 12, L729 (1979).

17 J. L. Cardy, Random-field effects in site-disordered Ising antiferromagnets, Phys. Rev. B 29, 505 (1984).

18 N. Sourlas, Universality in random systems: the case of the $3 D$ random field Ising model, Comp. Phys. Comm. 121, 183 (1999).

19 A. K. Hartmann and U. Nowak, Universality in three dimensional random field systems, Eur. Phys. J. B 7, 105 (1999).

20 N. G. Fytas and V. Martin-Mayor, Universality in the Three-Dimensional Random-Field Ising Model, Phys. Rev. Lett. 110, 227201 (2013).

21 J. Machta, M. E. J. Newman, and L. B. Chayes, Replicaexchange algorithm and results for the three-dimensional random field Ising model, Phys. Rev. E 62, 8782 (2000).

22 M. E. J. Newman and G. T. Barkema, Monte Carlo Methods in Statistical Physics (Oxford University Press Inc., New York, USA, 1999).

23 H. G. Katzgraber, Introduction to Monte Carlo Methods (2009), (arXiv:0905.1629).

24 R. H. Swendsen and J. Wang, Replica Monte Carlo simulation of spin-glasses, Phys. Rev. Lett. 57, 2607 (1986).

25 C. Geyer, in 23rd Symposium on the Interface, edited by E. M. Keramidas (Interface Foundation, Fairfax Station, VA, 1991), p. 156.

${ }^{26}$ E. Marinari and G. Parisi, Simulated tempering: A new Monte Carlo scheme, Europhys. Lett. 19, 451 (1992).

27 J. J. Moreno, H. G. Katzgraber, and A. K. Hartmann, Finding low-temperature states with parallel tempering, simulated annealing and simple Monte Carlo, Int. J. Mod. Phys. C 14, 285 (2003).

28 A. P. Young and H. G. Katzgraber, Absence of an AlmeidaThouless line in Three-Dimensional Spin Glasses, Phys. Rev. Lett. 93, 207203 (2004).

29 Note that Ref. 21 contains a mistake regarding the definition of $n_{++}$and $n_{--}$.

${ }^{30}$ N. B. Wilding and A. D. Bruce, Density fluctuations and field mixing in the critical fluid, J. Phys. Cond. Mat. 4, 3087 (1992).
31 T. Jörg, H. G. Katzgraber, and F. Krzakala, Behavior of Ising Spin Glasses in a Magnetic Field, Phys. Rev. Lett. 100, 197202 (2008).

32 U. Wolff, Collective Monte Carlo updating for spin systems, Phys. Rev. Lett. 62, 361 (1989).

33 J. Esser, U. Nowak, and K. D. Usadel, Exact ground-state properties of disordered Ising systems, Phys. Rev. B 55, 5866 (1997).

34 A. K. Hartmann, Ground-state structure of diluted antiferromagnets and random field systems, Physica A 248, 1 (1998).

35 J. C. Picard and H. D. Ratliff, Minimum cuts and related problems, Networks 5, 357 (1975).

36 A. V. Goldberg and R. E. Tarjan, A new approach to the maximum-flow problem, J. ACM 35, 921 (1988).

37 K. Binder, Finite size scaling analysis of Ising model block distribution functions, Z. Phys. B 43, 119 (1981).

38 F. Cooper, B. Freedman, and D. Preston, Solving $\phi_{1,2}^{4}$ theory with Monte Carlo, Nucl. Phys. B 210, 210 (1982).

39 H. G. Ballesteros, A. Cruz, L. A. Fernandez, V. MartinMayor, J. Pech, J. J. Ruiz-Lorenzo, A. Tarancon, P. Tellez, C. L. Ullod, and C. Ungil, Critical behavior of the threedimensional Ising spin glass, Phys. Rev. B 62, 14237 (2000).

40 M. Palassini and S. Caracciolo, Universal Finite-Size Scaling Functions in the 3D Ising Spin Glass, Phys. Rev. Lett. 82, 5128 (1999).

41 H. G. Katzgraber, M. Körner, and A. P. Young, Universality in three-dimensional Ising spin glasses: A Monte Carlo study, Phys. Rev. B 73, 224432 (2006).

42 L. A. Fernandez, V. Martin-Mayor, and D. Yllanes, Critical behavior of the dilute antiferromagnet in a magnetic field, Phys. Rev. B 84, 100408 (2011).

43 A. Maiorano, V. Martin-Mayor, J. J. Ruiz-Lorenzo, and A. Tarancón, Weak first-order transition in the threedimensional site-diluted Ising antiferromagnet in a magnetic field, Phys. Rev. B 76, 064435 (2007).

44 A. T. Ogielski and D. A. Huse, Critical Behavior of the Three-Dimensional Dilute Ising Antiferromagnet in a Field, Phys. Rev. Lett. 56, 1298 (1986).

45 A. L. Talapov and H. W. J. Blöte, The magnetization of the 3D Ising model, J. Phys. A 29, 5727 (1996).

46 A. A. Middleton and D. S. Fisher, Three-dimensional random-field Ising magnet: Interfaces, scaling, and the nature of states, Phys. Rev. B 65, 134411 (2002).

47 B. Ahrens and A. K. Hartmann, Critical behavior of the random-field Ising model at and beyond the upper critical dimension, Phys. Rev. B 83, 014205 (2011).

48 A. K. Hartmann, Practical Guide to Computer Simulations (World Scientific, Singapore, 2009).

49 D. Yllanes, Ph.D. thesis, Universidad Complutense de Madrid (2011), (arXiv:cond-mat/1111.0266).

50 A. K. Hartmann and A. P. Young, Specific-Heat Exponent of Random-Field Systems via Ground-State Calculations, Phys. Rev. B 64, 214419 (2001).

51 N. G. Fytas and A. Malakis, Phase diagram of the 3D bimodal random-field Ising model, Eur. Phys. J. B 61, 111 (2008).

52 Z. Slanic, D. P. Belanger, and J. A. Fernandez-Baca, Scaling properties of the critical behaviour of the dilute antiferromagnet $\mathrm{Fe}_{0.93} Z_{n_{0.07}} F_{2}$, J. Phy. Cond. Mat. 13, 1711 (2001).

53 T. Jörg, Critical behavior of the three-dimensional bonddiluted Ising spin glass: Finite-size scaling functions and 
universality, Phys. Rev. B 73, 224431 (2006). 